\title{
Change Patterns for Model Creation: Investigating the Role of Nesting Depth ${ }^{\star}$
} (Position Paper)

\author{
Barbara Weber $^{1}$, Jakob Pinggera ${ }^{1}$, Victoria Torres ${ }^{2}$, and Manfred Reichert ${ }^{3}$ \\ 1 University of Innsbruck, Austria \\ \{barbara.weber, jakob.pinggera\}@uibk.ac.at \\ 2 Universitat Politècnica de València, Spain \\ vtorres@pros.upv.es \\ 3 University of Ulm, Germany \\ manfred.reichert@uni-ulm.de
}

\begin{abstract}
Process model quality has been an area of considerable research efforts. In this context, the correctness-by-construction principle of change patterns offers a promising perspective. However, using change patterns for model creation imposes a more structured way of modeling. While the process of process modeling (PPM) based on change primitives has been investigated, little is known about this process based on change patterns and factors that impact the cognitive complexity of pattern usage. Insights from the field of cognitive psychology as well as observations from a pilot study suggest that the nesting depth of the model to be created has a significant impact on cognitive complexity. This paper proposes a research design to test the impact of nesting depth on the cognitive complexity of change pattern usage in an experiment.
\end{abstract}

Keywords: Process Model Quality, Process of Process Modeling, Change Patterns, Exploratory Study, Problem Solving.

\section{Introduction}

Much conceptual, analytical, and empirical research has been conducted during the last decades to enhance our understanding of conceptual modeling. In particular, process models have gained significant importance due to their fundamental role for process-aware information systems. Even though it is well known that a good understanding of a process model has a direct and measurable impact on the success of any modeling initiative [1], process models display a wide range of quality problems impeding their comprehensibility and maintainability [2].

To improve process model quality, change patterns offer a promising perspective [3]. Instead of creating a process model using change primitives (e.g., add node, add edge) high-level change operations combining several change primitives are used as basic building blocks for model creation. Examples of change

\footnotetext{
^ This research is supported by Austrian Science Fund (FWF): P23699-N23.
} 
patterns include the insertion and deletion of process fragments or their embedding in loops. Particularly appealing is correctness-by-construction [3], i.e., the modeling environment provides only patterns to the process modelers, which ensure that a sound process model is transformed into another sound model.

The use of change patterns implies a different way of creating process models, since correctness-by-construction imposes a structured way of modeling by enforcing block structuredness. Irrespective of whether change patterns or change primitives are used, model creation requires process modelers to construct a mental model (i.e., internal representation) of the requirements to be captured in the process model [4]. In a subsequent step, the mental model is mapped to the constructs provided by the modeling language creating an external representation of the domain [4]. While the construction of the mental model presumably remains unaffected, the use of change patterns leads to different challenges concerning pattern selection and combination for creating the external representation. In particular, process modelers might have to look several steps ahead to construct a certain process fragment, which constitutes a major difference compared to the use of change primitives, which do not impose any structural restrictions.

The process of creating process models based on change primitives has caused significant attention leading to a stream of research on the process of process modeling (PPM) [4 7]. This research is characterized by its focus on the formalization phase of model creation, i.e., the modeler's interactions with the modeling environment [5]. Still, little is known about the PPM when utilizing change patterns. To fill this gap, we conducted a pilot study with 16 process modelers [8], which indicated that the cognitive complexity imposed by change pattern usage is highly related to the structure of the process model to be created, in particular the nesting depth of the model. Modelers did not face any major problems when constructing simple process fragments, e.g., when inserting activities in sequences, making an activity optional, or inserting an activity in parallel. Faced with more complex control flow structures, in turn, the structural restrictions imposed by change patterns led to considerable problems (i.e., detours or incorrect models). These observations were underlined by feedback of the participants who appreciate the correctness-by-construction guarantees, but feel restricted when faced with complex control flow constructs. To further investigate these observations this paper proposes a research design to test the influence of nesting depth on the cognitive complexity of change pattern use.

\section{Cognitive Foundations of Problem Solving}

We consider the creation of process models to be a complex problem solving task. Problem solving has been an area of vivid research in cognitive psychology for decades. Therefore, we turn to cognitive psychology to understand the processes followed by humans when solving a problem like creating a process model.

Schemata. The human brain contains specialized regions contributing different functionality to the process of solving complex problems. Long-term memory is responsible for permanently storing information and has an essentially unlimited 
capacity, while in working memory comparing, computing and reasoning take place [9]. Although the latter is the main working area of the brain, it can store only a limited amount of information, which is forgotten after 20-30 seconds if not refreshed [10]. The question arises how information can be processed with such limited capacity. The human mind organizes information in interconnected schemata rather than in isolation [9]. Those schemata, stored in long-term memory, incorporate general concepts of similar situations [9]. Whenever situations similar to a schema arise, the latter is retrieved to help organizing information by creating chunks of information that can be processed efficiently [11]. To illustrate how chunking actually influences the understandability of process models consider a fragment with one alternative branch. Unexperienced modelers may use three chunks to store such process: one for each XOR- gateway and one for the activity. In contrast, an expert may recognize the pattern for optional activities, i.e., a schema for optional activities is present in long-time memory, allowing the storage of the entire process fragment in one working memory slot.

Problem-Solving Strategies. Novices confronted with an unfamiliar problem cannot rely on specialized problem solving strategies. Instead, an initial skeletal plan is formed [12]. Then, they utilize general problem solving strategies, like means-ends analysis, due to the lack of more specific strategies for the task [13]. Means-ends analysis can be described as the continual comparison of the problem's current state with the desired end product. Based on this, the next steps are selected until a satisfying solution is found [13]. After applying the constructed plan, it can be stored in long-term memory as plan schema [12]. For this, task-specific details are removed from the plan schema resulting in a plan schema that can be automatically applied in similar situations [14]. When confronted with a problem solving task in the future, the appropriate plan schema is selected using case-based reasoning [15]. The retrieved plan schema provides the user with structured knowledge that drives the process of solving the problem [11, 15]. Plan schemata allow experts to decide what steps to apply to end up with the desired solution [16]. If the plan schema is well developed, an expert never reaches a dead end when solving the problem [17].

Plan schemata seem important when creating process models based on change patterns since patterns cannot be combined in an arbitrary manner, especially when complex control-flow structures have to be created. If no plan schema is available on how to combine patterns to create the desired process model, modelers have to utilize means-ends analysis until a satisfying solution is found. This behavior is more likely to result in detours and decreased modeling speed. Moreover, the cognitive complexity for conducting means-ends analysis increases when confronted with complex control-flow structures like deeply nested blocks, making it more difficult to reach the correct solution. In addition, respective structures require the modeler to possess schemata to process larger chunks of information beforehand (i.e., increased need for look-ahead) to be able to model the respective fragment fast and without any detours. As a consequence, mental effort increases as well as the probability for detours. 


\section{Research Design}

Based on the cognitive foundations in Sect. 2 we propose a research design to investigate the influence of nesting depth on the cognitive complexity of change patterns usage for model creation by means of a controlled experiment.

Subjects. As explained in Sect. 2] novices and experts differ in their problem solving strategies. While novices have to rely on general problem solving strategies like means-ends analysis, experts can rely on plan schemata. Since process modelers in practical settings are often not expert modelers, but rather casual modelers with a basic amount of training [18], we do not require modeling experts for our study. To avoid, however, that difficulties are caused by unfamiliarity with the tool, rather than by difficulties with the tasks themselves, we require subjects to be moderately familiar with process modeling as well as change patterns. For this, subjects are trained using theoretical backgrounds of change patterns, but also obtain hands-on experience in the creation of process models using change patterns to guarantee that they are sufficiently literate in change pattern usage. Regarding process modeling experience and experience in change patterns usage we assume a relatively homogeneous group, which is tested ex-post. Choosing subjects moderately familiar with process modeling and change patterns usage allows us to make statements about casual modelers that cannot be generalized to modeling experts.

Independent Variable and Factor Levels. As independent variable we consider the nesting depth of the solution model with factor levels: high and low.

Objects. As outlined in Sect. 1, the creation of a process model requires the process modelers to construct a mental model (i.e., internal representation) of the requirements to be captured in the process model [4] and to map this mental model to the constructs provided by the modeling language (i.e., creating an external representation of the domain [4]). Tasks should be designed such that it can be ensured that observed difficulties are caused by change patterns usage rather than problems understanding the domain and constructing the mental model (which would also exist when using change primitives for model creation). Therefore, to factor domain influences out, participating subjects are asked to re-model a process (denoted as reference model in the following) starting from an empty modeling canvas. For this, process designers have to apply a sequence of change patterns to incrementally re-construct the given reference model starting from the empty model canvas. In addition, activities are labeled A, B, C to reduce the potential impact of domain knowledge.

Since the experiment aims to compare the cognitive complexity of using change patterns depending on the nesting depth, two versions (with high and low nesting dept) of the modeling task have to be designed, making sure that both tasks differ only in their nesting depth and not in other model characteristics. From research into process model quality we know, for example, that the size of the process model impacts model comprehension [19] and that different control-flow constructs do not have the same cognitive complexity [20]. As a 
consequence, these factors have to be controlled when designing the material for the experiment. Our intention therefore is to choose 2 models with the same number of activities, the same change patterns, and the same minimum number of change patterns needed to construct the solution.

Response Variables. To operationalize cognitive complexity of change patterns usage we consider, (1) accuracy, i.e., how close the subject's solution is to the reference model, (2) efficiency, i.e., how many detours it takes them to reach the solution, (3) speed, i.e., how fast they create the solution, and (4) the required mental effort. To measure accuracy we consider product deviations, i.e., discrepancies between the modeler's solution and the reference model. For example, a process model which contains two product deviations, requires the application of two change patterns to transform that model into the reference model. To operationalize efficiency, we consider process deviations measuring the modeler's detours until coming up with the solution. Process deviations are calculated as difference between the number of applied change patterns to reach the solution and the minimum number of change patterns required for the task. Finally, we consider the time needed to accomplish the task (i.e., speed) as well as the required mental effort, measured using a questionnaire after the task [21].

Hypotheses. This leads us to the following null hypotheses.

- $H_{1,0}$ : High nesting depth does not lead to significantly more product deviations when compared to low nesting depth.

- $H_{2,0}$ : High nesting depth does not lead to significantly more process deviations when compared to low nesting depth.

- $H_{3,0}$ : High nesting depth does not require significantly more time when compared to low nesting depth.

- $H_{4,0}$ : High nesting depth does not impose a significantly higher mental effort when compared to low nesting depth.

Instrumentation. For data collection Cheetah Experimental Platform [22] is used, logging given answers (e.g., demographic data), the time to accomplish the tasks, and all model interactions to obtain process deviations.

Experimental Design. The experiment is conducted as balanced single factor experiment with repeated measurements. Prior to the experiment a familiarization phase takes place (i.e., subjects are trained using change patterns). Subjects are then randomly assigned to two groups of equal size, subsequently referred to as G1 and G2. To provide a balanced experiment with repeated measurements, the overall procedure consists of two runs. In the first run G1 applies factor level low nesting depth, G2 factor level high nesting depth. In the second run, factor levels are switched and G1 applies factor level high nesting depth, G2 factor level low nesting depth to the same object. Choosing such a cross design is an additional measure to counter potential learning effects. 


\section{Summary}

While the process of creating process models using change primitives has caused some interest in recent years [4 7], our understanding of the process of creating process models using change patterns is limited. This paper proposes a research design to investigate the PPM using change patterns in more detail. In particular, the impact of nesting depth on the cognitive complexity of creating models is examined. Results of the experiment will provide a better understanding of the PPM using change patterns and help to understand how to design tool-support for change patterns based modeling.

\section{References}

1. Kock, N., Verville, J., Danesh-Pajou, A., DeLuca, D.: Communication flow orientation in business process modeling and its effect on redesign success: Results from a field study. DSS 46, 562-575 (2009)

2. Mendling, J., Verbeek, H.M.W., van Dongen, B.F., van der Aalst, W.M.P., Neumann, G.: Detection and prediction of errors in EPCs of the SAP reference model. DKE 64, 312-329 (2008)

3. Weber, B., Reichert, M., Rinderle, S.: Change Patterns and Change Support Features - Enhancing Flexibility in Process-Aware Information Systems. DKE 66, 438-466 (2008)

4. Soffer, P., Kaner, M., Wand, Y.: Towards Understanding the Process of Process Modeling: Theoretical and Empirical Considerations. In: Daniel, F., Barkaoui, K., Dustdar, S. (eds.) BPM 2011 Workshops, Part I. LNBIP, vol. 99, pp. 357-369. Springer, Heidelberg (2012)

5. Pinggera, J., Zugal, S., Weidlich, M., Fahland, D., Weber, B., Mendling, J., Reijers, H.A.: Tracing the Process of Process Modeling with Modeling Phase Diagrams. In: Daniel, F., Barkaoui, K., Dustdar, S. (eds.) BPM 2011 Workshops, Part I. LNBIP, vol. 99, pp. 370-382. Springer, Heidelberg (2012)

6. Claes, J., Vanderfeesten, I., Reijers, H.A., Pinggera, J., Weidlich, M., Zugal, S., Fahland, D., Weber, B., Mendling, J., Poels, G.: Tying Process Model Quality to the Modeling Process: The Impact of Structuring, Movement, and Speed. In: Barros, A., Gal, A., Kindler, E. (eds.) BPM 2012. LNCS, vol. 7481, pp. 33-48. Springer, Heidelberg (2012)

7. Pinggera, J., Soffer, P., Zugal, S., Weber, B., Weidlich, M., Fahland, D., Reijers, H.A., Mendling, J.: Modeling Styles in Business Process Modeling. In: Bider, I., Halpin, T., Krogstie, J., Nurcan, S., Proper, E., Schmidt, R., Soffer, P., Wrycza, S. (eds.) EMMSAD 2012 and BPMDS 2012. LNBIP, vol. 113, pp. 151-166. Springer, Heidelberg (2012)

8. Weber, B., Pinggera, J., Torres Bosch, V., Reichert, M.: Change patterns in use: A critical evaluation. In: Proc. BPMDS (accepted, 2013)

9. Gray, P.: Psychology. Worth Publishers (2007)

10. Tracz, W.: Computer programming and the human thought process. Software: Practice and Experience 9, 127-137 (1979)

11. Jeffries, R., Turner, A., Polson, P., Atwood, M.: The Process Involved in Designing Software. In: Cognitive Skills and Their Acquisition, pp. 255-283. Erlbaum (1981)

12. Rist, R.: Schema Creation in Programming. Cognitive Science 13, 389-414 (1989) 
13. Kant, E., Newell, A.: Problem Solving Techniques for the design of algorithms. Information Processing \& Management 20, 97-118 (1984)

14. Anderson, J.: Acquisition of cognitive skill. Psychological Review 89, 369-406 (1982)

15. Guindon, R., Curtis, B.: Control of cognitive processes during software design: what tools are needed? In: Proc. CHI 1988, pp. 263-268 (1988)

16. Sweller, J.: Cognitive load during problem solving: Effects on learning. Cognitive Science, 257-285 (1988)

17. Brooks, R.: Towards a theory of the cognitive processes in computer programming. International Journal of Man-Machine Studies 9, 737-751 (1977)

18. Pinggera, J., Zugal, S., Weber, B., Fahland, D., Weidlich, M., Mendling, J., Reijers, H.A.: How the Structuring of Domain Knowledge Can Help Casual Process Modelers. In: Parsons, J., Saeki, M., Shoval, P., Woo, C., Wand, Y. (eds.) ER 2010. LNCS, vol. 6412, pp. 445-451. Springer, Heidelberg (2010)

19. Mendling, J., Strembeck, M., Recker, J.: Factors of process model comprehension - findings from a series of experiments. DSS 53, 195-206 (2012)

20. Figl, K., Laue, R.: Cognitive complexity in business process modeling. In: Mouratidis, H., Rolland, C. (eds.) CAiSE 2011. LNCS, vol. 6741, pp. 452-466. Springer, Heidelberg (2011)

21. Zugal, S., Pinggera, J., Reijers, H., Reichert, M., Weber, B.: Making the Case for Measuring Mental Effort. In: Proc. EESSMod 2012, pp. 37-42 (2012)

22. Pinggera, J., Zugal, S., Weber, B.: Investigating the Process of Process Modeling with Cheetah Experimental Platform. In: Proc. ER-POIS 2010, pp. 13-18 (2010) 